\title{
Using MALDI-TOF MS imaging and LC-HRMS for the investigation of the degradation of polycaprolactone diol exposed to different wastewater treatments
}

\author{
Daniel Rivas $^{1} \cdot$ Bozo Zonja $^{1} \cdot$ Peter Eichhorn $^{1} \cdot$ Antoni Ginebreda $^{1} \cdot$ Sandra $^{\text {Pérez }}{ }^{1}$. \\ Damià Barceló $^{1,2}$
}

Received: 8 March 2017 /Revised: 6 April 2017 / Accepted: 19 April 2017

(C) The Author(s) 2017. This article is an open access publication

\begin{abstract}
Polymers are used in high amounts in a wide range of applications from biomedicine to industry. Because of the growing awareness of the increasing amounts of plastic wastes in the aquatic environment during recent years, the evaluation of their biodegradability deserves special attention. In the past, most efforts were dedicated to studying the biodegradation of polyesters in soil and compost, while very little research has been conducted on their fate in wastewater. Here, we assessed the ability of bacterial communities residing in the aerobic and denitrification tank from a wastewater treatment plant (WWTP) to degrade the polymeric ester polycaprolactone diol (PCLD; average molecular weight of $1250 \mathrm{Da}$ ). Following the incubation of the solid polymer in WWTP tanks, matrixassisted laser desorption/ionization-mass spectrometry imaging (MALDI-MSI) was used to provide evidence for hydrolytic reactions and to study differences in the spatial degradation on the PCLD surface. It was demonstrated that regardless of the wastewater type, the chemical structure on the PCLD surface underwent modifications after 7 days of exposure.
\end{abstract}

Published in the topical collection Advances in LC-MS/MS Analysis with guest editors Damià Barceló and Mira Petrovic.

Electronic supplementary material The online version of this article (doi:10.1007/s00216-017-0371-1) contains supplementary material, which is available to authorized users.

Sandra Pérez

spsqam@cid.csic.es

1 Water and Soil Quality Research Group, Department of Environmental Chemistry, Institute of Environmental Assessment and Water Research (IDAEA-CSIC), Jordi Girona, 18-26, 08034 Barcelona, Spain

2 Catalan Institute of Water Research, Parc Científic i Tecnològic de la Universitat de Girona, c/Emili Grahit, 101, Edifici H2O, 17003 Girona, Spain
Apart from the parent PCLD peak series in MALDI-MSI mass spectra, the presence of a second oligomer series with mass peaks spaced by $m / z .114$ (as in PCLD) was observed. It was proposed to correspond to polycaprolactone ( $\mathrm{PCL}$ ) originating from the hydrolytic cleavage of the diethylene glycol from PCLD. Their ion masses were detected at $\mathrm{m} / z, 104$ below the PCLD peaks and their structures were proposed as PCL cyclized oligomers. Differences in the spatial distribution of low MW ions $(<800)$ between the aerobic and denitrifying exposed samples in MALDI MSI were also noticeable. While the ions at $\mathrm{m} / \mathrm{z}, 221.1,247.1$ and 449.2 predominated in the aerobic exposed sample, those at $m / z, 475.5$ and 677.4 were characteristic of the denitrifying one. The MALDI-MSI measurements in the low mass range were complemented with LC-HRMS analysis to determine plausible structures of the major degradation products. Ten transformation products (TPs) were detected in the denitrifying wastewater experiment, five of them were the result of ester hydrolysis forming caprolactone oligomers (TPs 220, 334, 448, 562, and 676) while the other series corresponded to formation of PCL chain with a terminal diethylene glycol, likewise formed by ester hydrolysis (TPs 246, 360, 474, 588, and 702).

Keywords MALDI-TOF MS imaging $\cdot$ Liquid chromatography high-resolution mass spectrometry .

Wastewater treatment plant · Polymer degradation

\section{Introduction}

Polycaprolactone diol (PCLD) is an aliphatic polyester in which two polycaprolactone (PCL) chains are linked via a diethylene glycol molecule. The low molecular weight PCLD is widely used in the manufacture of biodegradable polyurethanes $[1,2]$. From the point of view of stability, 
PCLD is known be to biodegradable making it attractive for specific applications in biomedicine, e.g., drug delivery, in medical devices, and as degradable sutures [3-5]. Recently, PCL-related polymers have been proved useful as carbon source and biofilm carrier for denitrifying microorganisms in tertiary wastewater treatments [6]. Thus, they can directly contribute to the release of synthetic polymers in wastewater treatment plants (WWTP). WWTPs essentially consist of a sequence of treatment steps designed to remove both organic matter and nutrients. Typically, they include a biological treatment under aerobic conditions designed to reduce organic load, followed by a tertiary treatment with nitrification/ denitrification process to remove ammonium and nitrate. Each treatment step is characterized by specific conditions (aerobic or anoxic), which result in the development of adapted microorganisms consortia [7]. It is well known that the degradation pathways and kinetics of synthetic organic compounds largely depend on the conditions (aerobic or anaerobic) under which the wastewater bacteria are striving. For instance, synthetic polyesters have been reported to display higher degradation rates under aerobic conditions than under anaerobic ones [6-9].

A plethora of mass spectrometric analytical techniques have been used for evaluating the degradation of synthetic polymers [10]. The main drawback of the techniques used to date (gas chromatography coupled to mass spectrometry (MS), liquid chromatography-MS, and matrix-assisted laser desorption/ionization time of flight MS (MALDI-ToF-MS) is the loss of spatial information that occurs in the preparation of a liquid sample (extract). This loss is due to the homogenization and dissolution of the sample, a step that can be obviated by employing MALDI-MS imaging (MALDI MSI) on the intact solid sample. By two-dimensional scanning of the sample surface with the laser beam following deposition of a suitable matrix, analyte ions are being desorbed from the surface and extracted into the ToF analyzer for mass spectral recording. Due to the large $m / z$ operating range of the ToFMS, high molecular weight components of the polymer under investigation can easily be studied. Characterization of polymers [11], polymeric dialyzer membranes [12], and polymers used for joint implants [13] have been performed by MALDIToF-MSI. However, the accuracy of MALDI average molecular weights has often been discussed. When MALDI-ToFMSI is compared to high-resolution MS (HRMS) instruments commonly used in environmental laboratories for identification of small molecules, e.g., Orbitrap-MS [14], it presents limited MS/MS capability relative to its lower mass resolving power and mass accuracy [15].

Several studies have evaluated the biodegradability of polymers in soil, compost [6], wastewaters [16], and marine environments [17]; however, less attention has been paid to the biodegradation of polyesters in wastewaters. Recently, we performed a comparative study on the degradation of a polyester, PCLD, in two aquatic environments, a river and a laboratory mesocosm, applying MALDI-ToF-MS [18]. In a follow-up study [19], we explored the capability of MALDI-MSI as an analytical tool for investigating modifications in the chemical composition at the polymer surface after exposure to either sterile, aerobic, or denitrifying conditions, using PCLD as a probe polymer. Whereas the acquisition of the MALDI mass spectral data and the subsequent image processing proved a powerful technique for studying 2D spatial degradation patterns of the polymer surface, its mass resolution capability turned out to be insufficient for assigning chemical structures to the mass peaks attributed to a series of emerging transformation products [20]. This limitation prompted us to undertake a third study of PCLD degradation exploiting in parallel the spatial capabilities of MALDI-MSI and the identification power of LC-HRMS [11].

The objective of the present study was to investigate the degradation of PCLD following exposure to wastewater bacteria in the aerobic and denitrifying reactor tanks of a municipal WWTP. We set out to identify the chemical changes in the polymer structure leading to degradation products and to determine their spatial distribution on the surface. This was accomplished by making use of LC-HRMS and MALDI-MSI, respectively.

\section{Materials and methods}

\section{Chemical reagents and materials}

Polycaprolactone diol homopolymer (average MW = $1250 \mathrm{Da}$ ) used in the degradation experiments was purchased from Polysciences (Warrington, PA, USA). The matrix trans-2-[3-(4tert-butylphenyl)-2-methyl-2-propenylidene] malononitrile (DCTB) was purchased from Aldrich (Steinheim, Germany). PCLD was placed in the capsule units (Lancaster, UK) used as polymer holders in the biodegradation experiments; they were purchased from Exposmeter AB (Tavelsjo, Sweden). Each capsule unit consisted of a plastic piston and a plastic cap with a window $2 \mathrm{~cm}$ in diameter (see Electronic Supplementary Material (ESM), Fig. S1). Acetonitrile and water were purchased from Fisher Scientific (Geel, Belgium). Formic acid (98-100\%) was ACS grade and purchased from Sigma-Aldrich (Schnelldorf, Germany).

\section{Degradation experiments}

\section{Wastewater treatment plant description}

The wastewater generated by the southern district of Barcelona reaches the WWTP located in Prat de Llobregat (see ESM, Fig. S2) through several pumping stations after a pretreatment for the elimination of sand and grease it passes through an activated sludge biological treatment plant. About $60 \%$ of the treated water is discharged into the sea through 
Table 1 High mass range (MW > $800 \mathrm{Da}$ ) transformation product structures identified by in the different polymer samples studied. Structures on the right column are those of the starting polycaprolactone diol (PCLD) polymer, while those in the left column correspond to polycaprolactone (PCL) polymer formed as transformation product under denitrifying conditions

\begin{tabular}{|c|c|c|c|}
\hline \multirow[t]{2}{*}{$\mathrm{m} / \mathrm{z}$ of $[\mathrm{M}+\mathrm{Na}]^{+}$} & \multirow[t]{2}{*}{$n+1$} & $m / z$ of $[\mathrm{M}+\mathrm{Na}]^{+}$ & $n+m$ \\
\hline & & 813.5 & 6 \\
\hline 822.6 & 7 & 927.5 & 7 \\
\hline 937.4 & 8 & 1041.6 & 8 \\
\hline 1051.6 & 9 & 1155.7 & 9 \\
\hline 1165.2 & 10 & 1269.7 & 10 \\
\hline 1278.3 & 11 & 1383.8 & 11 \\
\hline 1393.2 & 12 & 1497.9 & 12 \\
\hline 1506.6 & 13 & 1611.9 & 13 \\
\hline 1621.0 & 14 & 1726.0 & 14 \\
\hline 1736.0 & 15 & 1840.1 & 15 \\
\hline 1850.1 & 16 & 1954.1 & 16 \\
\hline 1963.3 & 17 & 2068.2 & 17 \\
\hline 2077.7 & 18 & 2182.3 & 18 \\
\hline 2192.2 & 19 & 2296.3 & 19 \\
\hline 2306.5 & 20 & 2411.2 & 20 \\
\hline 2420.9 & 21 & 2524.9 & 21 \\
\hline 2536.8 & 22 & 2640.8 & 22 \\
\hline 2650.2 & 23 & 2754.2 & 23 \\
\hline
\end{tabular}

$3.2 \mathrm{~km}$ long emissary. The remaining $40 \%$ is subjected to tertiary treatment for nutrient removal and subsequent reuse (environmental flow and irrigation). Main technical characteristics of the plant are reported in the ESM, Table S1. 
Table 2 Low mass transformation product structures identified by either LC-HRMS, MALDI MSI or both in the different polycaprolactone diol samples studied

\begin{tabular}{|c|c|c|c|c|c|c|}
\hline $\begin{array}{l}m / z \text { of } \\
{[\mathrm{M}+\mathrm{H}]^{+}}\end{array}$ & Error & $\begin{array}{l}\text { Detected } \\
\text { by LC- } \\
\text { HRMS }\end{array}$ & $\begin{array}{l}\text { Detected } \\
\text { by MALDI- } \\
\text { MSI }\end{array}$ & $\begin{array}{l}\text { Sterile } \\
\text { control }\end{array}$ & $\begin{array}{l}\text { Aerobic } \\
\text { wastewater }\end{array}$ & $\begin{array}{l}\text { Denitrifying } \\
\text { wastewater }\end{array}$ \\
\hline 221.1382 & -0.7 & $X$ & $X$ & & $\mathrm{X}$ & $X$ \\
\hline 247.1538 & -0.7 & $\mathrm{X}$ & $X$ & & $X$ & $X$ \\
\hline \multirow[t]{2}{*}{335.2059} & \multirow[t]{2}{*}{-1.7} & \multirow[t]{2}{*}{$X(2)^{\mathrm{a}}$} & \multirow[t]{2}{*}{$X$} & & $\mathrm{X}$ & $\mathrm{X}$ \\
\hline & & & & & $X$ & $\mathrm{X}$ \\
\hline 361.2214 & -1.8 & $\mathrm{X}$ & & & $\mathrm{X}$ & $\mathrm{X}$ \\
\hline \multirow[t]{2}{*}{449.2738} & \multirow[t]{2}{*}{-1.6} & \multirow[t]{2}{*}{$X(2)^{\mathrm{a}}$} & \multirow[t]{2}{*}{$X$} & & $\mathrm{X}$ & $X$ \\
\hline & & & & & $X$ & $\mathrm{X}$ \\
\hline 475.2897 & -1.0 & $\mathrm{X}$ & $X$ & & $\mathrm{X}$ & $X$ \\
\hline \multirow[t]{3}{*}{563.3420} & \multirow[t]{3}{*}{-1.0} & \multirow[t]{3}{*}{$X(3)^{\mathrm{a}}$} & \multirow[t]{3}{*}{$X$} & & $\mathrm{X}$ & $\mathrm{X}$ \\
\hline & & & & & $\mathrm{X}$ & $\mathrm{X}$ \\
\hline & & & & & $\mathrm{X}$ & $\mathrm{X}$ \\
\hline 589.3577 & -0.9 & $\mathrm{X}$ & & & $X$ & $X$ \\
\hline \multirow[t]{3}{*}{677.4101} & \multirow[t]{3}{*}{-0.8} & \multirow[t]{3}{*}{$X(3)^{\mathrm{a}}$} & \multirow[t]{3}{*}{$X$} & & & $\mathrm{X}$ \\
\hline & & & & & & $\mathrm{X}$ \\
\hline & & & & & & $X$ \\
\hline 703.4255 & -1.1 & $X$ & & & $\mathrm{X}$ & $\mathrm{X}$ \\
\hline
\end{tabular}

${ }^{\mathrm{a}}$ Number of peaks

\section{Degradation experiments in WWTP}

Degradation experiments were carried out by placing three capsules filled with PCLD (3-6 g) in the secondary (aerobic) and denitrifying reactors. Samples were collected after 7 days of exposure and analyzed as explained below. Moreover, the following parameters were routinely monitored as part of the regular in-process operational WWTP control: suspended solid, BOD, COD, nitrates, nitrites, phosphor, nitrogen, conductivity, and $\mathrm{pH}$. Data corresponding to the days in which the experiment took place are given (see ESM, Table S1).

\section{Control experiments at lab scale}

For control and comparison purposes, a parallel blank experiment was carried out in the lab, in which capsules containing the polymer were exposed to sterilized water at room temperature during the same period of time (7 days). Furthermore, a polymer sample standard (i.e., non-exposed) was analyzed as explained below.

\section{MALDI MSI}

\section{Sample preparation}

For MALDI-TOF-MS measurements, the polymer was removed from the capsules and dried in a dessicator for $15 \mathrm{~min}$. Slices of 12-16 $\mu \mathrm{m}$ were cut in a cryo-microtome (Leica CM3050S, Nußloch, Germany) and placed onto indium-tin oxide (ITO)- coated glass slides (Sigma-Aldrich). The samples were placed in a desiccator for $15 \mathrm{~min}$ before matrix deposition. The section was coated with $30 \mathrm{mg}$ of DCTB using a sublimation device (Fisher Scientific, Madrid, Spain). Sublimation conditions were $100 \mathrm{mTorr}$ pressure at $140^{\circ} \mathrm{C}$ during $15 \mathrm{~min}$. The sample slice was externally cooled with ice.

\section{Mass spectrometry}

All experiments were performed using an AuToFlex III MALDI-TOF/TOF instrument (Bruker Daltonik GmbH, Bremen, Germany) equipped with a Smartbeam laser at $200 \mathrm{~Hz}$ laser at the "medium focus" setting and were controlled using FlexControl 3.0 (Bruker Daltonik GmbH). The analytical conditions for the MALDI MSI analysis were set as follows: positive reflector ion mode; ion source 1 voltage, $19 \mathrm{kV}$; ion source 2 voltage, $16 \mathrm{kV}$; lens voltage, $8.5 \mathrm{kV}$; and mass range $\mathrm{m} / \mathrm{z}, 100-3000$. Laser irradiance was maintained slightly above threshold. Mass spectra were acquired with spatial resolution set at $200 \mu \mathrm{m}$. For each pixel, 250 laser shots were measured per position as a sum of 25 consecutive laser shots in 10 random walk shot steps.

\section{Image processing}

Raw spectra were analyzed with Flex Imaging 4.0 software (Bruker Daltonik GmbH, Bremen, Germany) after baseline subtraction and root mean square (RMS) normalization. Ion density 
maps were created for ions observed from the skyline projection spectrum. Masses were manually selected with a mass accuracy set to $\pm 0.1 \%$ (manual peak picking). Regions of interest were manually defined by using both the sample image and MSI data. Image processing and data analysis were done using SCiLS Lab software (Bremen, Germany) allowing identification and quantification of the altered areas.

\section{LC-HRMS}

Clean cuts of the polymer exposed in wastewater tanks at the WWTP were dissolved in methanol and then diluted with water (95:5 water:methanol) to achieve the initial concentration of $1 \mathrm{mgL}^{-1}$ of the polymer. The samples were injected directly $(10 \mu \mathrm{L})$ into LC-HRMS. A generic LC-HRMS method was applied to detect and identify TPs of the PCLD using an Acquity UPLC coupled to Q-Exactive (Thermo Scientific, Bremen, Germany). Electrospray ionization (ESI) interface was operated in both positive and negative ionization mode to obtain the exact masses of the TPs. The chromatographic separation was performed using an Acquity UPLC $\mathrm{C}_{18}$ column $(100 \times 2.1 \mathrm{~mm}, 1.7 \mu \mathrm{m})$ proceeded by a pre-column of the same packing material $(5 \times 2.1 \mathrm{~mm}, 1.7 \mu \mathrm{m})$. The mobile phases for the chromatographic run were as follows: (a) acetonitrile with $0.1 \%$ formic acid and (b) water $(0.1 \%$ formic acid). The total chromatographic run was 15 min with a flow rate of $300 \mu \mathrm{L} \mathrm{min}^{-1}$. The elution was accomplished with the following solvent gradient: $0 \mathrm{~min}(10 \% \mathrm{~A})-1 \mathrm{~min}(10 \% \mathrm{~A})-$ $11 \min (95 \% \mathrm{~A})$ and $13 \min (95 \% \mathrm{~A})-13.5 \mathrm{~min}(10 \% \mathrm{~A})$ and stabilized until $15 \mathrm{~min}$. The column temperature was set to $40{ }^{\circ} \mathrm{C}$. For the detection method, a data dependent scan was applied with the following parameters: full scan and datadependant $\mathrm{MS}^{2}$ were set to resolution of 35,000 [full width at half maximum (FWHM)], with the full scan range of $m / z$ 100-1000. In the data-dependent scan, the isolation window was $m / z, 2$ and the normalized collision energy was (NCE)
Fig. 1 Average MALDI-ToF mass spectra recorded in the positive reflectron mode, showing a polycaprolactone diol series $[\mathrm{M}+\mathrm{Na}]^{+}$in the range from $\mathrm{m} / \mathrm{z}, 23$ to 2600 . The differences in the intensity scales: (A) standard polycaprolactone diol; (B) after 7 days of exposure in sterile water. (C) After 7 days of exposure in water under aerobic conditions (WWTP secondary treatment tank); (D) after 7 days of exposure in water under denitrifying conditions (WWTP tertiary treatment tank).
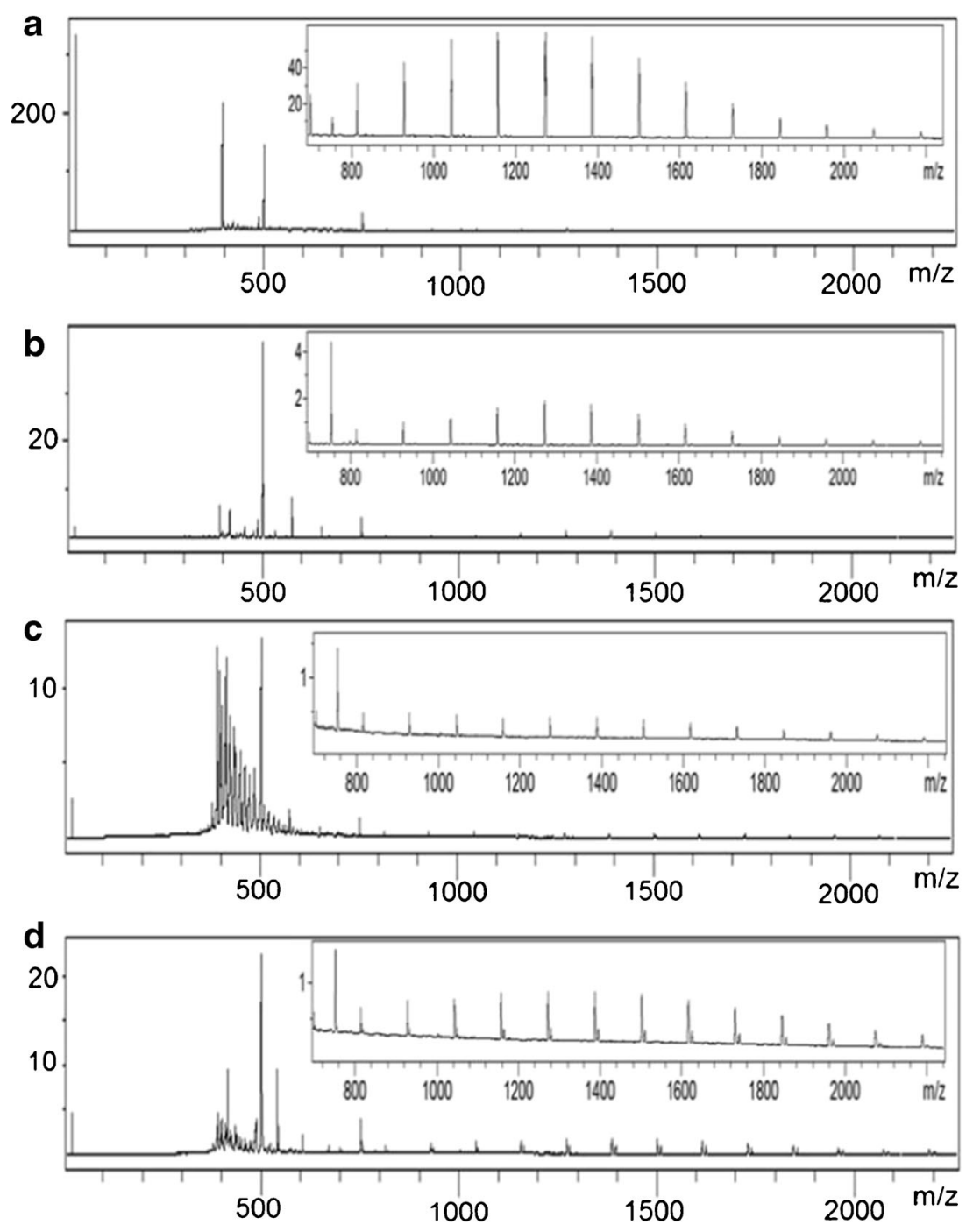
Fig. 2 Proposed degradation pathway of polycaprolactone diol: (A) formation of polycaprolactone (PCL) linear oligomers; (B) formation of cyclic oligomers and $(\mathbf{C})$ formation of polycaprolactone-diethylene glycol monoester

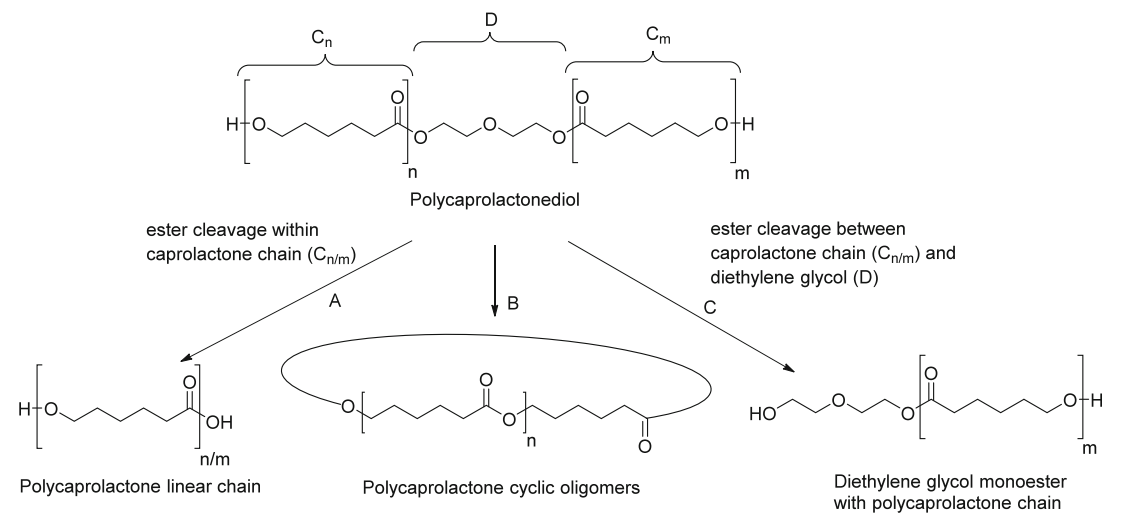

either 15 or 35 . Both positive and negative ionization mode runs were used and then compared to a blank sample (containing only the parent homopolymer dissolved in pure water and methanol (95:5)) with the degraded samples using SIEVE 2.2 (Thermo Fisher).

\section{Results and discussion}

\section{MALDI mass spectra of polymer sections}

Representative average MALDI-TOF mass spectra (mass range up to $\mathrm{m} / \mathrm{z}$ 2600) of sections of the starting material, the sterile control, and the two samples exposed to aerobic and denitrifying wastewater are shown in Fig. 1A-D at different attenuation factors. Highest mass range $(\mathrm{m} / \mathrm{z}, 700-2600)$ is consistent with the starting polymer structure with increasing number of monomer (caprolactone) units (peak spacing $\mathrm{m} / \mathrm{z}$ 114). Overall, there is a general decrease of the relative peak intensities in the exposed samples (Fig. 1B-D) as well as differences in the peak distribution, thus suggesting a different distribution of the chain lengths.

This trend is more pronounced in the samples exposed to aerobic and denitrifying conditions (Fig. 1C, D). In addition, in the spectrum of the latter sample (Fig. 1D), the presence of a second series of oligomers, likewise, spaced by $m / z, 114$ (i.e., $\mathrm{m} / \mathrm{z} 1051.6$ and 1165.6) and separated from the homolog peaks of the main series by $m / z$ 104. It was attributed to PCL (Table 1) formally corresponding to a loss of the diethylenglycol unit from PCLD polymer and they were identified as PCL cyclic oligomers [21-24], previously reported as a possible degradation pathway of PCL (Fig. 1) [25].

As regards the lower mass range series $(\mathrm{m} / \mathrm{z}<800)$, with the peak at $\mathrm{m} / \mathrm{z}, 500$ corresponding to the matrix DCTB, there is also evidence in the degraded polymer samples (Fig. 1C-D) of peaks attributable to degradation of the standard material as well. Again, this is detected in the polymer exposed to aerobic and denitrifying conditions. The structural identification of these peaks was further investigated in detail using LCHRMS (see "Identification of transformation products using
LC-HRMS"). Overall, the above findings are indicative of degradation processes affecting the exposed samples. This is consistent with the fact that polyesters and specifically polycaprolactones are reported to undergo degradation under both aerobic and anaerobic conditions [6].

\section{Identification of transformation products using LC-HRMS}

Since there was no qualitative difference between the runs in the positive and negative ionization mode, transformation products (TPs) were identified using the positive ESI. For the identification of polymer TPs, the product ion spectra of the TPs were recorded to determine the most likely elemental compositions and to identify structures of the fragment ions.

All compounds detected were formed as the result of ester hydrolysis of the parent homopolymer (Fig. 2). In total, ten TPs were detected and identified in LC-HRMS (see ESM, Tables S1 and S2). Five were the result of ester hydrolysis forming caprolactone oligomers (see ESM, Table S1 in the homologous series TPs 220, 334, 448, 562, and 676) while the other series detected corresponded to formation of PCL chain with a terminal diethylene glycol (see ESM, Table S2), via (again) ester hydrolysis (TPs 246, 360, 474, 588, and 702). In all cases, the difference between the polymer series was the repeating unit of $\mathrm{m} / \mathrm{z} 114.0681$ (6-hydroxyhexanecarbonyl, $\left.\mathrm{HOCH}_{2}-\left(\mathrm{CH}_{2}\right)_{5}-\mathrm{CO}-\right)$ (see ESM, Fig. S3). In general, all TPs were predominantly detected as ionized sodium adducts and $[\mathrm{M}+\mathrm{H}]+$.

The fragmentation of TP 220 started with a water loss followed by loss of $m / z 44.0262\left(\mathrm{C}_{2} \mathrm{H}_{4} \mathrm{O}\right)$. Its homolog series followed the same pattern (see ESM, Table S1). As for the other series, their fragmentation pattern again was the water loss in combination with loss of $\mathrm{C}_{2} \mathrm{H}_{4} \mathrm{O}$, after the initial water loss from the acid itself. Detailed fragmentation pattern and mass spectra can be found in the ESM on Tables S1 and S2. A summary of the main peaks identified in "MALDI mass spectra of polymer sections" and "Identification of transformation products using LC-HRMS," together with their proposed structure is given in Tables 1 and 2 for low mass range 
(MW < $800 \mathrm{Da})$ and high mass range $(\mathrm{MW}>800 \mathrm{Da}) \mathrm{TPs}$, respectively.

UPLC-HRMS chromatograms corresponding to the low mass range TPs of the denitrifying exposed sample are shown in Fig. 3. Peaks corresponding to polycaprolactone (PCL) fragments (Fig. 3A; TPs 246, 360, 474, 588, and 702) are single as expected from the hydrolysis pattern, while those of polycaprolactone-diethylene glycol monoesters (Fig. 3B: TPs $220,334,448,562$, and 676) appear resolved totally or partially in more than one peak (Table 2), tentatively pointing to the presence of isomer mixtures. However, their HRMS-(ESI)mass spectra (Fig. 4) cannot confirm this hypothesis.
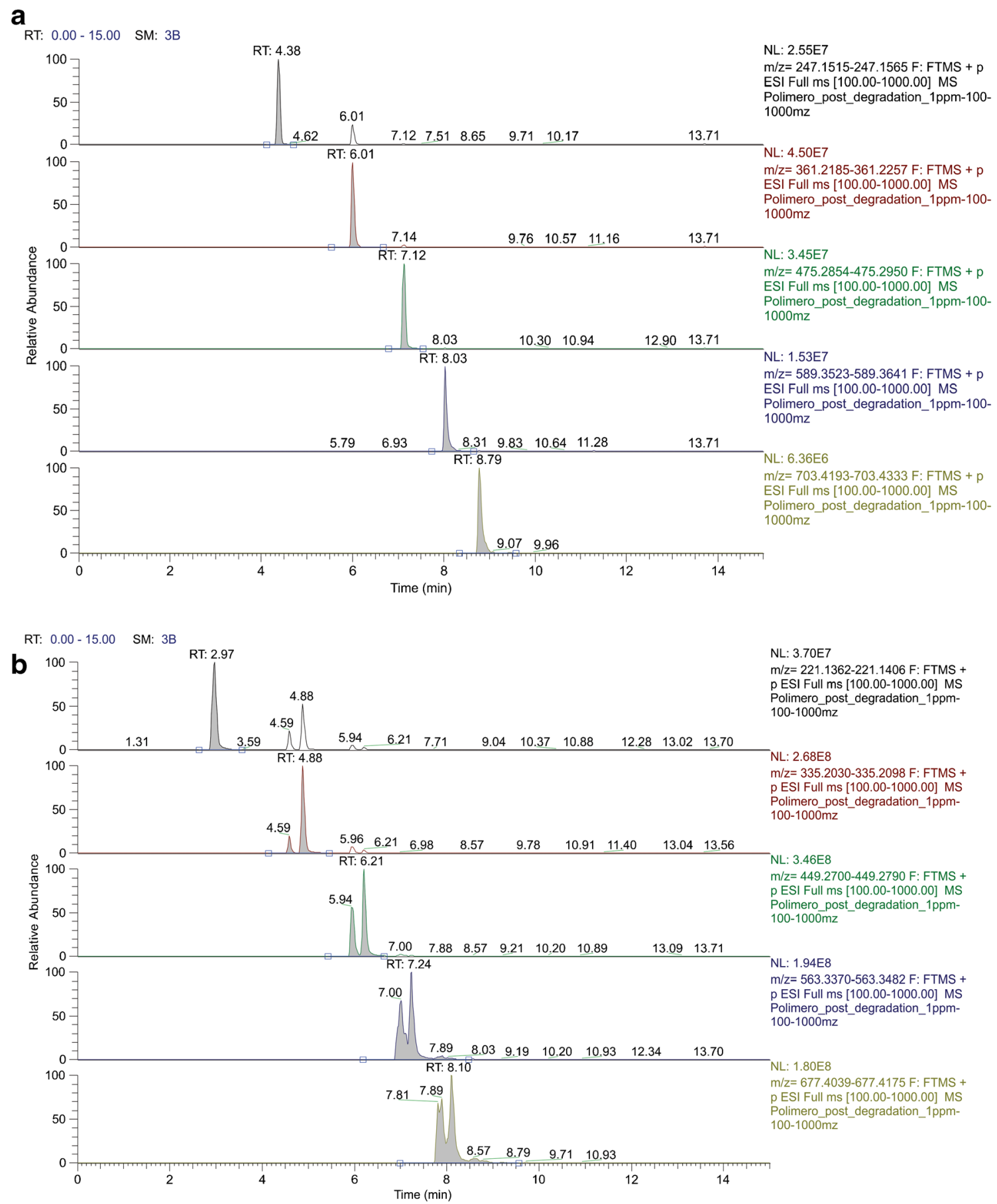

Fig. 3 UPLC-HRMS chromatogram of the TPs in the low mass range $(\mathrm{m} / \mathrm{z}<800)$; (A) formed by ester hydrolysis and (B) formation of polycaprolactone-diethylene glycol monoester in degradation sample at $t 7$ days (see also Fig. 2) 


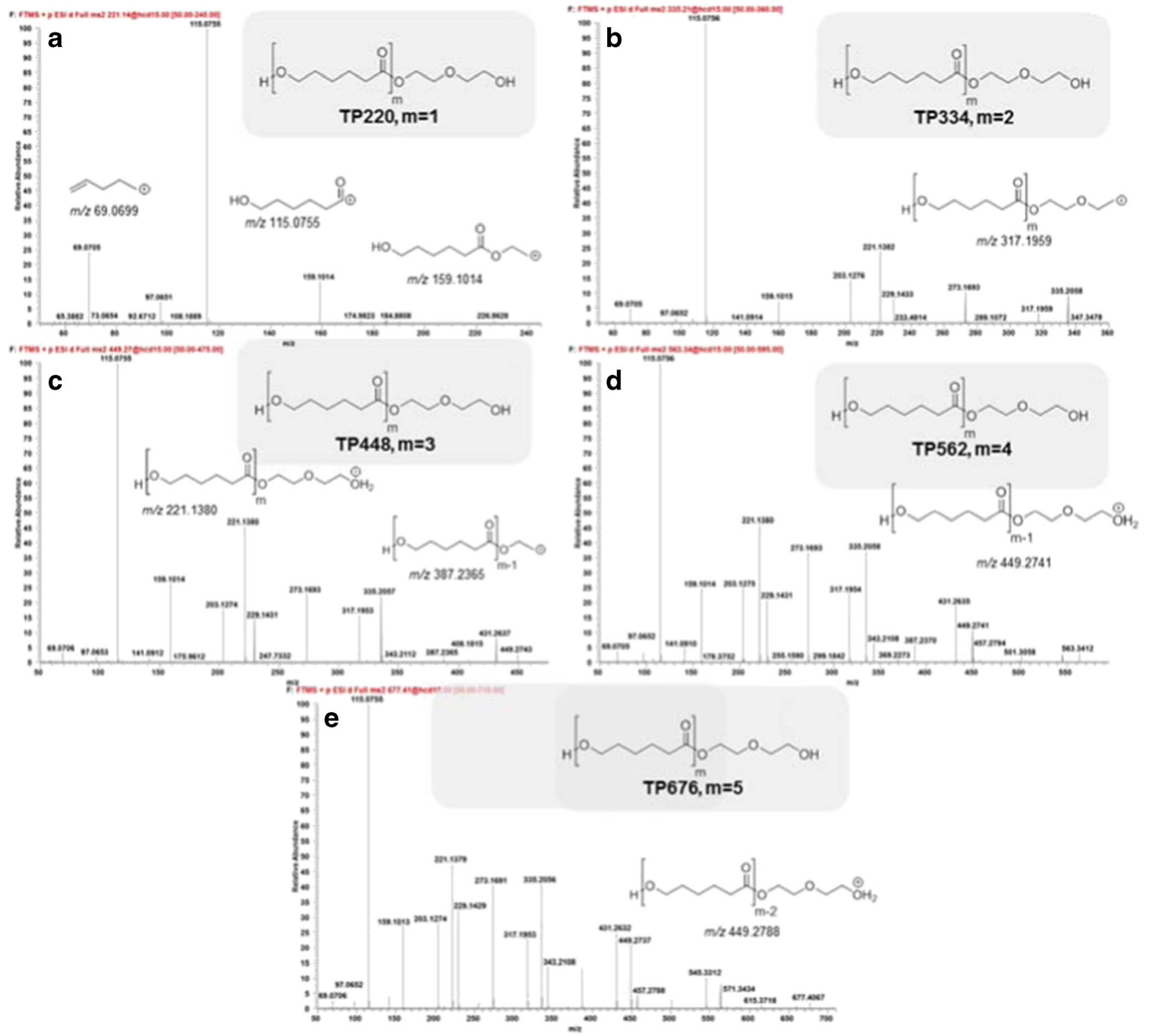

Fig. 4 HRMS-(ESI)-mass spectra of (A) TP220, (B) TP334, (C) TP448, (D) TP562, and (E) TP676

\section{Spatial distribution of polymer degradation patterns by MALDI MSI}

Biotic-mediated processes taking place in the surface of polymer samples exposed in the WWTP environment were expected to exhibit large spatial heterogeneity, especially if they are associated to the formation of biofilm colonies in the surface of the exposed material. This fact should be correspondingly reflected in the surface composition pattern of the exposed polymer probes. To investigate it, MALDI MSI appears as a suitable technique since it allows generating 2D images across the probe surface using selected ions. In Fig. 5A-D, MALDI MSI images generated from polymer cuts of the four samples analyzed using the main ions characteristic of the starting polymer $(\mathrm{m} / \mathrm{z} 1155.7,1269.7,1383.8$, and 1497.9) are shown as an example. They exhibit notable differences in the spatial distribution on both intensity and uniformity (homogeneity). Whereas the standard polymer (Fig. 5A) shows a highly uniform and intense distribution, exposed samples (Fig. 5B-D) deploy increasingly heterogeneous spatial patterns with a decreasing intensity in the order sterile->aerobic->denitrifying. In the latter case (Fig. 5D), the disappearance of the peak monitored is almost complete in some parts (deep blue colored areas). These findings are fully consistent with those derived from the average MALDI MS exposed in "MALDI mass spectra of polymer sections."

The spatial pattern of second oligomer series predominantly formed in the denitrifying exposed sample was investigated 
Fig. 5 Comparison of MALDIToF images corresponding to sections of (A) PCDL standard, (B) control sample kept in sterilized water, $(\mathbf{C})$ sample exposed to aerobic wastewater, and (D) sample exposed to denitrifying wastewater. Images were obtained by data processing with Flex Imaging 4.0 software. Spatial differences among pixels using the different ions are visible across the surface of the samples. Ions shown are sodium adducts $[\mathrm{M}+\mathrm{Na}]^{+}$

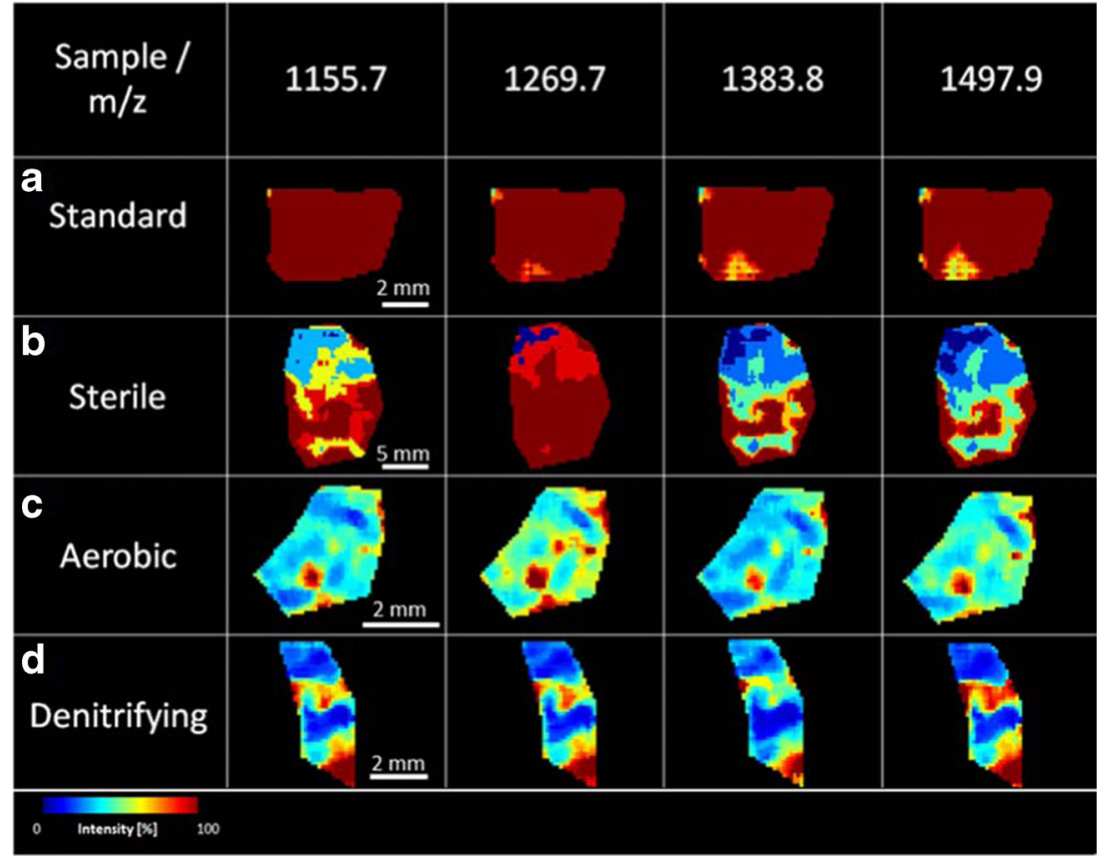

using MALDI MSI as well. The images corresponding to the main parent oligomer and the second series formed by hydrolytic loss of the diethylene glycol moiety are shown in Fig. 5. In each compartment, the transformed polymer peak is shown on the right side while the formally parent peak is on the left side.
Their MW differs on $m / z$ 104, which corresponds to the diethylene glycol fragment lost. The images corresponding to the main parent oligomer and the second series formed by internal "back-biting" cyclization are shown in Fig. 6. In each compartment, the cycled analog ion distribution is shown on the left side
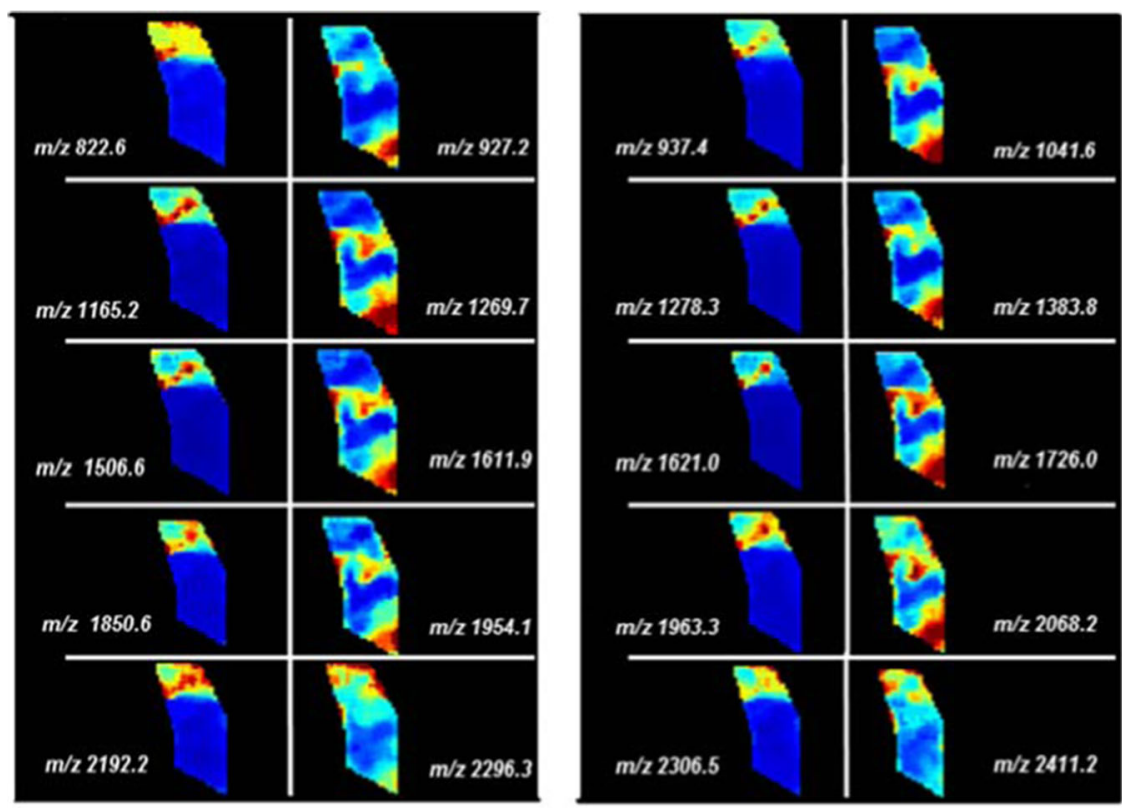

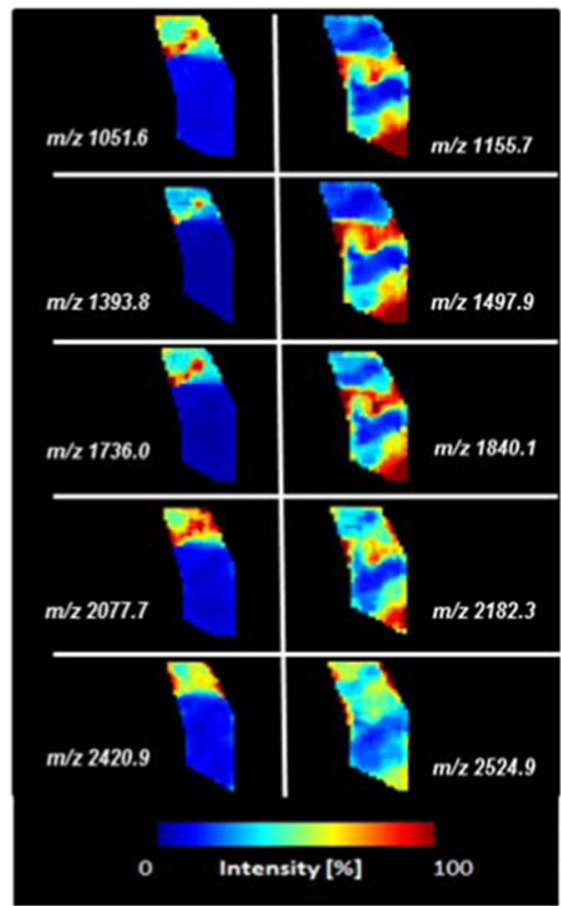

shown corresponds (right) to the peaks of the starting polycaprolactone diol polymer PCDL (M) and (left) the cyclic polycaprolactone analog (M-104). Ions shown are sodium adducts $[\mathrm{M}+\mathrm{Na}]^{+}$
Fig. 6 Comparison of MALDI-ToF images corresponding to sections of samples exposed to denitrifying wastewater. Images were obtained by data processing with Flex Imaging 4.0 software. Each pair of images 
Fig. 7 Comparison of MALDIToF images corresponding to sections of samples exposed to aerobic (left) and denitrifying (right) wastewater using characteristic ions $(\mathrm{m} / \mathrm{z}<800)$. Images were obtained by data processing with Flex Imaging 4.0 software.

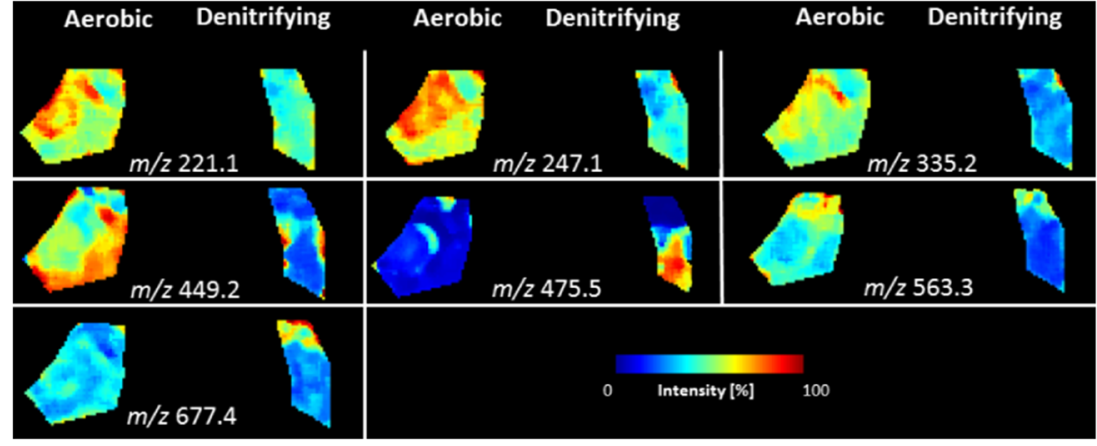

while the formally parent peak ion distribution is on the right side. It is worth noting that the transformation polymer is highly unevenly distributed across the sample, appearing mostly located in the upper part and it is roughly complementary to the starting polymer. Such pattern is approximately repeated for all peaks shown in Fig. 6.

We finally studied the spatial distribution of low MW range ions (i.e., below m/e 700). Their structures are reported in Table 2. Most of them were confirmed by LC-HRMS as explained above. Comparing the aerobic and denitrifying exposed sample images, some differences are perceptible. Whereas ions $m / z, 221.1,247.1$, and 449.2 seem to predominate in the aerobic exposed sample, those at $\mathrm{m} / \mathrm{z} 475.5$ and 677.4 are characteristic for the denitrifying exposed sample. Remarkably, while the surface distribution of the latter one $(\mathrm{m} /$ $z$ 677.4) follows a similar spatial pattern than those observed for the aforementioned transformation polymer (Fig. Fig. 7, right side images, upper part), the former $(\mathrm{m} / \mathrm{z}$ 475.5) fits the opposite and complementary distribution (Fig. Fig. 7, right side images, lower part).

\section{Conclusions}

This study has been demonstrated the complementarity of the MSI and UPLC-HRMS techniques for the determination of the biodegradation products of polycaprolactone diol. Whereas UPLC-HRMS has higher sensitivity thus allowing the unequivocal identification of degradation products through their exact masses, MALDI MSI technique shows how these degradation products are distributed across the surface of the exposed sample.

In addition, different transformation products of the starting polycaprolactone diol oligomer were identified in both the low and high molecular weight mass ranges. On the other hand, there is evidence of three different degradation processes as well, namely (a) the hydrolytic cleavage of the diethylenglycol-polycaproic ester bond, (b) another one taking place on the polyester bonds of the polycaprolactone units, and (c) the cyclization of polycaprolactone oligomers via internal ester formation. The former one seems specific of the denitrifying exposed samples. On the other hand, there is evidence of two different ester hydrolysis processes as well, namely the cleavage of the diethylenglycol-polycaproic ester bond and another one taking place on the polyester bonds of the polycaprolactone moiety. The former one seems specific of the denitrifying exposed samples. Both processes were traced through the corresponding degradation products.

Finally, the study of MALDI MSI images reveal the spatial complexity and specificity of the degradation processes taking place in the exposed polymer surface, which allow clearly differentiation between aerobic and denitrifying environments as found in conventional engineered systems. Such differential behavior is better highlighted in comparison with a sample kept under sterile conditions. Whereas abiotic processes seem to be present as well, it clearly supports the contribution of biotic processes acting on the aerobic and denitrifying exposed samples.

Acknowledgements This study has been financially supported by the EU through the FP7 project GLOBAQUA (Grant Agreement No 603629), and by the Generalitat de Catalunya (Consolidated Research Groups "2014 SGR 418-Water and Soil Quality Unit" and 2014 SGR 291-ICRA). It reflects only the author's views. The Community is not liable for any use that may be made of the information contained therein.

\section{Compliance with ethical standards}

Conflict of interest The authors declare that they have no conflict of interest.

Open Access This article is distributed under the terms of the Creative Commons Attribution 4.0 International License (http:// creativecommons.org/licenses/by/4.0/), which permits unrestricted use, distribution, and reproduction in any medium, provided you give appropriate credit to the original author(s) and the source, provide a link to the Creative Commons license, and indicate if changes were made.

\section{References}

1. Rattanapan S, Pasetto P, Pilard JF, Tanrattanakul V. Preparation and properties of bio-based polyurethane foams from natural rubber and polycaprolactone diol. J Polym Res. 2016;23(9) doi:10.1007/ s10965-016-1081-7. 
2. Nadjiadjim G, Sheibat-Othman N, Févotte G, Dupuy J. Development and monitoring of a continuous polyurethane pilot reactor. Chem Eng Technol. 2010;33(11):1900-8. doi:10.1002/ ceat.201000296.

3. Tehrani AD, Parsamanesh M. Preparation, characterization and drug delivery study of a novel nanobiopolymeric multidrug delivery system. Mater Sci Eng C. 2017;73:516-24.

4. Schek RM, Taboas JM, Segvich SJ, Hollister SJ, Krebsbach PH. Engineered osteochondral grafts using biphasic composite solid freeform fabricated scaffolds. Tissue Eng. 2004;10(9-10):1376-85.

5. Munj HR, Lannutti JJ, Tomasko DL. Understanding drug release from PCL/gelatin electrospun blends. J Biomater Appl. 2016;31:933-49.

6. Eubeler JP, Bernhard M, Knepper TP. Environmental biodegradation of synthetic polymers II. Biodegradation of different polymer groups. TrAC Trends Anal Chem. 2010;29(1):84-100.

7. Wang J, Chu L. Biological nitrate removal from water and wastewater by solid-phase denitrification process. Biotechnol Adv. 2016;34(6):1103-12.

8. Li P, Zuo J, Wang Y, Zhao J, Tang L, Li Z. Tertiary nitrogen removal for municipal wastewater using a solid-phase denitrifying biofilter with polycaprolactone as the carbon source and filtration medium. Water Res. 2016;93:74-83.

9. Abou-Zeid D-M, Müller R-J, Deckwer W-D. Biodegradation of aliphatic homopolyesters and aliphatic-aromatic copolyesters by anaerobic microorganisms. Biomacromolecules. 2004;5(5):1687-97.

10. Rizzarelli P, Carroccio S. Modern mass spectrometry in the characterization and degradation of biodegradable polymers. Anal Chim Acta. 2014;808:18-43. doi:10.1016/j.aca.2013.11.001.

11. Weidner SM, Falkenhagen J. Imaging mass spectrometry for examining localization of polymeric composition in matrix-assisted laser desorption/ionization samples. Rapid Commun Mass Spectrom. 2009;23(5):653-60. doi:10.1002/rcm.3919.

12. Krueger K, Terne C, Werner C, Freudenberg U, Jankowski V, Zidek W, Jankowski J. Characterization of polymer membranes by MALDI mass-spectrometric imaging techniques. Anal Chem. 2013;85(10):4998-5004. doi:10.1021/ac4002063.

13. Fröhlich SM, Archodoulaki V-M, Allmaier G, MarchettiDeschmann M. MALDI-TOF mass spectrometry imaging reveals molecular level changes in ultrahigh molecular weight polyethylene joint implants in correlation with lipid adsorption. Anal Chem. 2014;86(19):9723-32. doi:10.1021/ac5025232.

14. Trim PJ, Snel MF. Small molecule MALDI MS imaging: current technologies and future challenges. Methods. 2016;104:127-41. doi:10.1016/j.ymeth.2016.01.011.

15. Verhaert PDEM, Pinkse MWH, Strupat K, Conaway MCP. Imaging of similar mass neuropeptides in neuronal tissue by enhanced resolution MALDI MS with an ion trap - OrbitrapTM hybrid instrument. In: Rubakhin SS, Sweedler JV, editors. Mass spectrometry imaging: principles and protocols. Totowa: Humana Press; 2010. p. 433-49. doi:10.1007/978-1-60761-746-4_25.

16. Otal E, Mantzavinos D, Delgado MV, Hellenbrand R, Lebrato J, Metcalfe IS, Livingston AG. Integrated wet air oxidation and biological treatment of polyethylene glycol-containing wastewaters. J Chem Technol Biotechnol. 1997;70(2):147-56. doi:10.1002/(SICI) 1097-4660(199710)70:2\&lt;147::AID-JCTB747\&gt;3.0.CO;2-3.

17. Staples CA, Williams JB, Craig GR, Roberts KM. Fate, effects and potential environmental risks of ethylene glycol: a review. Chemosphere. 2001;43(3):377-83.

18. Rivas D, Ginebreda A, Pérez S, Quero C, Barceló D. MALDI-TOF MS imaging evidences spatial differences in the degradation of solid polycaprolactone diol in water under aerobic and denitrifying conditions. Sci Total Environ. 2016;566-567:27-33.

19. Rivas D, Ginebreda A, Elosegi A, Pozo J, Pérez S, Quero C, Barceló D. Using a polymer probe characterized by MALDITOF/MS to assess river ecosystem functioning: from polymer selection to field tests. Sci Total Environ. 2016;573:532-40.

20. Dopstadt J, Vens-Cappell S, Neubauer L, Tudzynski P, Cramer B, Dreisewerd K, Humpf H-U (2016) Localization of ergot alkaloids in sclerotia of Claviceps purpurea by matrix-assisted laser desorption/ ionization mass spectrometry imaging. Anal Bioanal Chem :1-10.

21. Kricheldorf HR, Langanke D, Stricker A, Räder HJ. Polylactones, 53: formation of cyclic polyesters in the combined ring-expansion polymerization/ring-opening polycondensation of lactones. Macromol Chem Phys. 2002;203(2):405-12. doi:10.1002/15213935(20020101)203:2\&1t;405::AID-MACP405\&gt;3.0.CO;2-N.

22. Kricheldorf HR, Stricker A, Langanke D. Polylactones, 52. Tin carboxylates as initiators of $\varepsilon$-caprolactone. Macromol Chem Phys. 2001;202(15):2963-70.

23. Báez JE, Martínez-Richa A, Marcos-Fernández A. One-step route to $\alpha$-hydroxyl- $\omega$-(carboxylic acid) polylactones using catalysis by decamolybdate anion. Macromolecules. 2005;38(5):1599-608. doi:10.1021/ma0491098.

24. Ahmed H, Trathnigg B, Oliver Kappe C, Saf R. Characterization of poly(ethylene glycol)-b-poly( $\varepsilon$-caprolactone) by liquid chromatography under critical conditions: influence of catalysts and reaction conditions on product composition. Eur Polym J. 2009;45(8):233847. doi:10.1016/j.eurpolymj.2009.05.001.

25. Bankova M, Kumar A, Impallomeni G, Ballistreri A, Gross RA. Mass-selective lipase-catalyzed poly( $\varepsilon$-caprolactone) transesterification reactions. Macromolecules. 2002;35(18):685866. doi:10.1021/ma0202282. 